\title{
EL CUERPO AUDIOVISUALIZADO EN LA REALIZACIÓN DEL VÍDEO MUSICAL CONTEMPORÁNEO
}

\author{
THE AUDIO-VISUALIZED BODY IN THE MAKING OF \\ THE CONTEMPORARY MUSICAL VIDEO
}

\author{
ANA SEDEÑO-VALDELLÓS*, VIRGINIA GUARINOS**
}

RESUMEN: Este trabajo de investigación se enmarca, de un modo interdisciplinar, entre los estudios del cuerpo, la tecnología y los discursos audiovisuales. Se centra en el análisis del videoclip musical como formato/género. Dentro de él, los trabajos sobre corporeidad se han centrado tradicionalmente en la representación del cuerpo humano como elemento expuesto a la mirada, como objeto de consumo visual integrante de una puesta en escena, pero rara vez como un elemento en el complejo entramado que componen realización, tecnología y sonido, como parte de los productos mainstream. En ese sentido, existen, aun no siendo comunes, una serie de técnicas y recursos audiovisuales que son puestos en práctica en numerosos vídeos musicales para componer fórmulas de síncresis músico-visual. En definitiva, en el artículo se analiza un corpus de videoclips a través del análisis textual, para tratar de localizar las técnicas de realización y de representación espacio-temporal, que permiten la audiovisualización de la presencia corporal de los miembros del grupo, con el objetivo de incrementar una identificación y fruición espectatorial: imitación y analogía, audiovisualización de parámetros musicales, texto escrito y juegos con el espacio son algunos de estos recursos del cuerpo audiovisualizado en el videoclip.

Palabras Clave: Videoclip musical; teoría del cuerpo; realización audiovisual; síncresis músicovisual; técnicas de postproducción visual.

Aвstract: This research work is developed within a interdisciplinary framework among the studies of the body, technology and audiovisual discourses. It focuses on the analysis of the music video as format and genre. In this genre, works on corporeality have traditionally focused on the representation of the human body as an element exposed to the gaze, as an object of visual consumption that is part of a staging, but rarely as an element in the complex framework that make up filmmaking, technology and sound, as part of mainstream products. In this sense, there are, although not common, a series of audiovisual techniques and resources that are put into practice in numerous

\footnotetext{
* Doctora en Comunicación Audiovisual. Académica de la Universidad de Málaga, Málaga, España. Correo electrónico: valdellos@uma.es. Orcid: https://orcid.org/0000-0003-3897-2457

** Doctora en Comunicación Audiovisual. Académica de la Universidad de Sevilla, Sevilla, España. Correo electrónico: guarinos@us.es. Orcid: https://orcid.org/0000-0002-7270-0087
} 
music videos to compose formulas for music-visual syncrisis. In sum, the article analyzes a corpus of music video through textual analysis, trying to identify the techniques of realization and spatial-temporal representation, which allow the audiovisual presentation of the body presence of the members of the group, with the aim of increasing identification and enjoyment in the spectators: imitation and analogy, audiovisual visualization of musical parameters, texts and games with space are some of these resources of the audio-visualized body in the contemporary music video.

Keywords: Music Video, Body Theory; Audiovisual Production; Music-Visual Synchronization; Technics of Audiovisual Postproduction.

Recibido: 12.06.2019. Aceptado: 08.07.2020.

\section{INTRODUCCIÓN}

$\mathrm{C}$

omo texto cultural central de la contemporaneidad, el videoclip musical recibe importantes influencias por la interconexión de contenidos que permite. Debido a que probablemente es el formato más consumido en redes sociales (gracias a plataformas de vídeo como YouTube, donde la publicidad y todo tipo de contenido se metamorfosean para llegar a audiencias diferenciadas) ha conformado una especie de formato-tipo, que es retomado -o plagiado- en duración y presentación de materias audiovisuales por otros neoformatos publicitarios como el fashion-film, el branded-content o la vídeo-noticia. Naturalmente, esto resulta coherente con la tendencia crossmedia de todos los mensajes culturales, que es la extrema hibridación de procesos y materiales gracias a su transferencia o transformación en el mismo tipo de datos o inputs digitales, es decir, gracias a la flexibilidad y maleabilidad técnica que permite la edición del material sonoro, visual y textual, tal como Nicolas Bourriaud (2009) describe con su concepto de postproducción.

Estas habilidades modificadoras de los procesos de producción y edición de imagen y sonido parecen no tener límites, abren las fronteras de lo posible, y conducen a una virtualización completa de la realidad, siguiente paso después de la digitalización. Esto se puede describir como el reinado de la imagen interfaz, que destaca como punto intermedio entre el mundo analógico -conocido- y el fluido visual de la realidad virtual, como utopía -o distopía- en lo que la estética de la falsedad de lo posible se asienta.

Un videoclip musical es un formato audiovisual que supone un mensaje muy codificado y fruto de una alta hibridación cultural y mediática. Por un lado, todo videoclip es un formato publicitario que pretende vender un producto musical. La música es una construcción cultural generada por el 
ser humano, lo que lo sitúa en el centro de la visualización en su momento creativo musical, ya sea como cantante, músico o figurante del videoclip. Por otro lado, el cuerpo del artista es su soporte físico y su representación resulta imprescindible en estos discursos audiovisuales musicales, bien de modo real o simulado (como sucede en algunos videoclips oficiales en los que son dobles o alter egos los que imitan a los cantantes) o de animación; en cualquiera de los casos, siempre aparecerá un cuerpo humano representando al cantante o a los personajes vinculados a la letra de la canción o la historia narrada por la banda de imágenes visuales.

Este conjunto de música y cuerpos, de relatos, representaciones y puesta en escena, es recogido, a modo de refuerzo semántico y, muchas veces también de refuerzo rítmico, o a modo de reinterpretación y experimentación visual, por una realización, que es, en sí misma, otro cuerpo físico, el del propio videoclip.

\section{FUNDAMENTOS TEÓRICOS Y METODOLÓGICOS}

Si algo define transversalmente las prácticas culturales contemporáneas, es su insistencia en el cuerpo humano como campo de reflexión, como referencia y como objeto artístico. Los estudios sobre el cuerpo tuvieron una época de asentamiento de reflexiones y multiplicidad de publicaciones en la década de los noventa del siglo XX y en la primera del nuevo milenio, especialmente desde los marcos filosóficos, literarios, de los estudios culturales y la antropología (Turner, 1994). Desde Goffman (1981), en 1959, hasta la revolución de la perspectiva queer de Judith Butler (1993), la originaria perspectiva sociológica ha ido ampliándose con un resurgir de los estudios sobre el cuerpo desde las ciencias sociales y humanidades bajo el prisma de los estudios culturales y las teorías feministas, que han hecho ampliarse y especializarse este tipo de trabajos de investigación hasta acercarlos a los productos audiovisuales (Shilling, 2007).

Antropología y corporeidad (Aguado, 2004), tecnología y cultura corporal (Mendiola y Arpal, 2007), el cuerpo en la sociedad mediática (Lucerga, 2004), el cuerpo en televisión (Gordillo y Guarinos, 2010), y un largo listado de estudios del cuerpo femenino en el arte, la literatura, la publicidad, se acercan al posicionamiento de este trabajo, relacionado con la metodología de cuestionamiento de los paradigmas epistemológicos empleados en el análisis de la imagen visual para el estudio de la relación entre el sujeto espectador y el objeto mirado, que en este caso es el cuerpo humano, 
compartiendo la idea de Villota (2004), sobre la desaparición simbólica de dos cuerpos, uno expuesto a la mirada y otro observador, generando una única entidad, que llama el investigador imagen-cuerpo, en la que el cuerpo del espectador y la imagen del cuerpo observado se constituyen mutuamente en el acto en sí de la mirada.

En este sentido, el artículo pretende demostrar que esto que sucede con la imagen-cuerpo se produce por medio del dispositivo tecnológico que media entre ellos, y la realización audiovisual, de forma específica sobre el videoclip musical. Se parte de la idea de que, efectivamente,

el cuerpo ha resultado el objeto y "el centro de muchas preocupaciones tecnológicas e ideológicas. En la producción, en el consumo, en el ocio, en los espectáculos, en la publicidad, etc., el cuerpo se ha convertido en un objeto de tratamiento, de manipulación, de mise-en-scene, de mercadeo. Sobre el cuerpo convergen todo tipo de intereses sociales y políticos en la actual "civilización tecnológica". (Brohm, como se citó en Bernard, 1976, p. 13)

Como parte de esta civilización tecnológica, el videoclip musical, un objeto preferente de consumo entre la población internacional más joven, el cuerpo se cosifica, especialmente, se fragmenta y dispone a la mirada, se utiliza e instrumentaliza con objetivos sexuales, ideológicos y políticos. Se produce con ello una pérdida de su organicidad, lo que concluye en un permanente juego con la identidad de artistas, intérpretes y músicos, rasgo fundacional de la música popular industrial o mainstream (Guarinos, 2012). Como ya se ha descrito en anteriores publicaciones (Sedeño-Valdellós, 2012, Vernallis, 1998), el videoclip dirige su realización en esta dirección, abriendo su interpretación a lo político.

Sin embargo, en este texto se trabaja en otra dirección. Se presentan diversos casos que ponen el foco en cómo se modula la señal icónica como recurso de realización, de manera que se evidencien diversos parámetros sonoro-musicales de la canción, con un uso de lo corporal que se distancia de la mayoría de los videoclips musicales. No se trata de vídeos marginales, sino de artistas reconocidos y comerciales, lo que supone una excepción a la norma. Por ello, se plantea como hipótesis que la audiovisualización de rasgos musicales (estructura, ritmo, forma melódica) encamina el proceso creativo del realizador/director, potenciando la puesta en escena performance, con la que se formaliza o encarna visualmente la identidad del músico/intérprete. Esta cualidad parece definir el videoclip musical mainstream o industrial actual, que se beneficia de unas técnicas de postpro- 
ducción digital sin límites en lo relacionado con la edición de la imagen, aunque no es nueva para el videoclip, como bien argumentó Goodwin: "Mi punto de vista es que los espectáculos visuales (baile, gesto, la exhibición de virtuosismo, iluminación, bombas de humo, hielo seco, retroproyección, etc.) han trabajado siempre en conjunto con la música en sî" (Goodwin, 1992, p. 18, trad. propia).

De hecho, Alf Bjornberg argumenta que es la cualidad de sintaxis elíptica de la música popular la que condiciona las estructuras visuales de la banda icónica del clip: "Las características distintivas del vídeo musical pueden explicarse mejor a partir de la comprensión de las características sintácticas de la música popular que a través de las extendidas teorías postmodernistas" (Bjornberg, 1994, p. 51). En la misma línea, Gillet, Essid y Richard (2007) y Korsgaard (2012) también describen el potencial visualizador del cuerpo en el videoclip bajo el término de modulación de señal, como una nueva forma de producir analogías musicovisuales entre impresiones sensoriales.

En este sentido, la realización de un videoclip musical se basa en la interrelación musicovisual, que conecta un discurso visual creado para el tema musical que se promociona. En resumen, se pretenden describir modelos y técnicas de sincronía estética (Rodríguez, 1998) o síncresis (Chion, 1993) en el videoclip, "coincidencia exacta en el tiempo de dos estímulos distintos que el receptor percibe perfectamente diferenciados. Estos dos estímulos pueden ser percibidos por el mismo sentido o por sentidos distintos" (Rodríguez, 1998, p. 253). Son "la soldadura irresistible y espontánea que se produce entre un fenómeno sonoro y un fenómeno visual momentáneo cuando estos coinciden en un mismo momento, independientemente de toda lógica racional" (Chion, 1993, p. 65). La tarea del realizador en la creación de un videoclip es la búsqueda de técnicas de unión entre las formas sonoras y las visuales que conduzcan a esa síncresis. Una forma de materializar esa unión musicovisual es visualizar por imitación, aunque "el trabajo del director tiene un carácter fundamentalmente creativo y, en consecuencia, no se trata de un mero trabajo de traducción visual o un ejercicio puramente técnico" (Selva, 2012, p. 108).

También Gaudin habla de los efectos de lo musicovisual sobre la identidad de los músicos en términos de amplificación o intensificación de la representación: "Su efecto más obvio es la amplificación, mediante la movilización de todos los medios visuales y sonoros de la composición, de la idea sensible de la mecanización del cuerpo. (...) inscrito en la materia musical-visual de la obra, este efecto de mecanización llega directamente a nuestro cuerpo de audio-espectadores" (Gaudin, 2018, pp. 103-104). 
Para Mairs-Slee (2016) esta conexión es la esperada en términos de experiencia física, destacándola en el caso de los videoclips con danza:

El movimiento, la danza y la acción corporeizada sirven como elemento constituyente del video musical que puede mover al oyente / espectador, desde un consumo pasivo a través de los oídos y los ojos a una participación activa a través del cuerpo. $(2016$, p. 147)

A partir de estos fundamentos y bajo la hipótesis formulada, el objetivo trazado es la localización de las técnicas con las que se produce esta tendencia, en la que la performance se amplifica o revitaliza: si la puesta en escena performance se basa en la actuación en forma de concierto o simulación del mismo, al modo televisivo, ahora se construyen puntos sincrónicos de encuentro musicovisual con técnicas de realización y postproducción. A través de estas se produce una integración participativa de los cuerpos mediados, del performance, con el sonido y la canción.

Se han seleccionado como corpus 20 videoclips. La muestra es intencional, razonada a los objetivos de reflexión y análisis de este aspecto elegido, conjugada con un conocimiento sobre el panorama del videoclip contemporáneo. Algunos trabajos de investigación previos habían detectado tendencias que se analizan y explicitan en este estudio, aunque como la muestra es intencional se debe relativizar la validez de los resultados. Como afirma Gaytán,

la sociología del cuerpo y de las emociones debe situarse en una posición de nominalismo moderado, en tanto que asumimos que la realidad se construye a partir de marcos de sentido distintos para cada entorno cultural, aunque compartidos socialmente y, en esa medida, que son elementos subjetivos objetivables. (2010, s/p)

Las herramientas metodológicas empleadas se encuadran en el análisis textual. Concretamente, se han estudiado:

-Las técnicas básicas de realización y su aplicación en la relación discurso visual-discurso musical, como el encuadre, tipo y cambio de plano y su movimiento, que suponen una ilustración activa de la canción como materia sonora, es decir, como discurso con parámetros de ritmo, velocidad y textura musical, etc.

-Las técnicas avanzadas de realización, como las herramientas de postproducción o de grabación no simples (cámaras súper lentas, stop motion) 
y las de composición y tratamiento de imagen (layering, VFX, CGI, Motion Graphics...). En esta categoría se incluyen fórmulas ya empleadas con las que la realización se aproximaba o imitaba procedimientos de las vanguardias artísticas (en su búsqueda utópica), de lo que luego se denominó música visual. Esta vertiente de la creación audiovisual contemporánea ha desarrollado técnicas como la de los videojockeys, el videomapping o el livecoding, etc. Todas esta sprácticas han desarrollado software, aplicaciones y plugins específicos muy diversos, que han permitido un control detallado de los parámetros de la imagen en función de la señal de sonido, utilizando los espectogramas de audio, los sintetizadores y los visualizadores de formas de onda y FFT.

-La forma básica de sincronía o relación preferente entre la música y lo visual. En este criterio podemos diferenciar (Simeon, 1992) entre correspondencias o acuerdos como la correspondencia kinética, la correspondencia sintagmática, y la correspondencia de contenido. La primera se produce respecto del ritmo o pulso de la imagen y está en videoclips con danza o performance. La segunda, sintagmática, recoge la correspondencia estructural que viene desde la canción y tiene que ver con cambios de sección (estribillo, estrofa...). La tercera, correspondencia de contenido, se refiere a alusiones directas en la banda visual respecto a lo sonoro, en cuanto a las letras y a su uso como texto visual.

\section{RESULTADOS}

Las estrategias de interacción musicovisual derivadas de la realización audiovisual sobre los cuerpos audiovisualizados en los videoclips musicales pueden agruparse en función de los siguientes parámetros, relacionados con el ritmo, la imitación visual de lo musical, el reflejo o integración de la letra de la canción y la construcción de una espacialidad evocadora.

Ritmo, velocidad y frecuencia.

El primero de los parámetros claramente idóneo en esta tarea es el ritmo, con el que se pueden establecer correspondencias kinéticas, entre velocidad de la música y velocidad de la acción en la puesta en escena y/o montaje. El modo básico de esta correspondencia, que establece tipos de analogía música/imagen, se produce entre cambios de plano/ritmo básico o tempo. Vernallis habla de verticalidad rítmica (2004) o cut on the beat, para des- 
cribir las imágenes que están editadas según el tempo musical. También Rafols y Colomer (2006) explican estas analogías rítmicas, que provienen del terreno de la música en el cine:

La música de base rítmica y de percusión rápida crea una fuerte tensión y reclama mucho movimiento de elementos diferentes. La música de base melódica con notas largas sin demasiados cambios y sin percusión reclama movimientos más suaves, una imagen de mayores matices y unos colores poco saturados. (p. 35)

Fórmulas menos frecuentes de correspondencias kinéticas resultan de la imitación de técnicas de edición sonora como el sampling (extracción y/o emulación electrónica de un fragmento sonoro de cualquier tipo), el breakbeat (extracción de un fragmento sonoro y su introducción en otro contexto y repetición hasta generar un nuevo segmento musical) o el scratching (fricción adelante y atrás de la púa del tocadiscos con el vinilo). Pueden verse en canciones o piezas musicales de hip-hop. En Welcome to heartbreaker $^{1}$ de Kayne West se percibe un interés por demostrar un apego a la repetición visual de determinados momentos instrumentales, a modo de golpes percusivos. La impresión visual que este efecto deja consiste en un parpadeo de aparición de una segunda capa sobre la principal, que mantiene mayor duración para los planos sobre el cantante, y la desestabilización del plano.

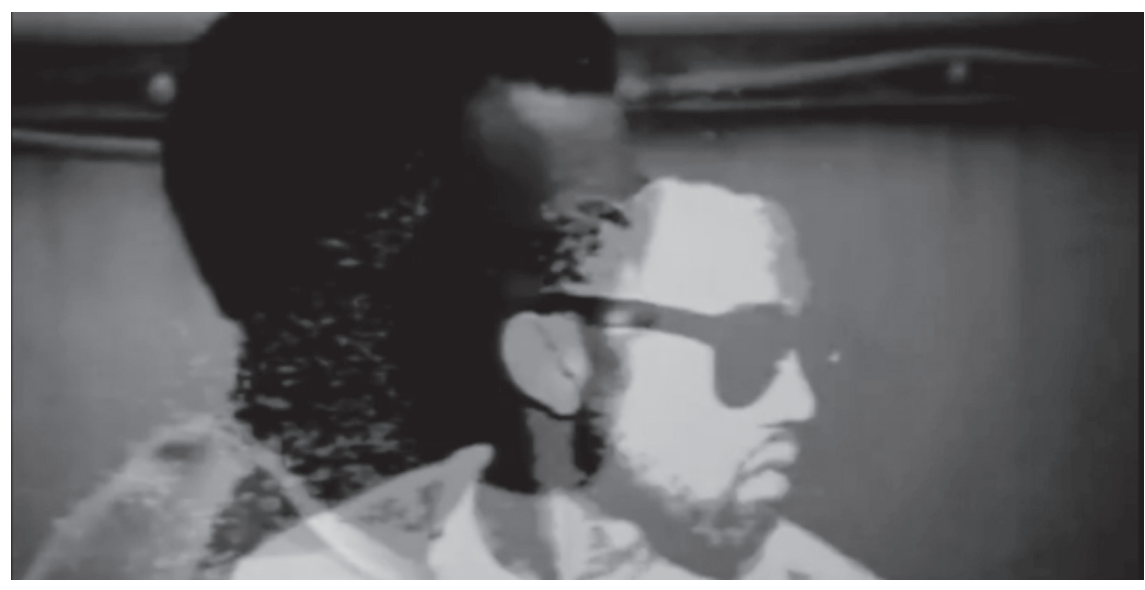

Figura 1. Welcome to heartbreak (Kayne West, 2013).

\footnotetext{
${ }^{1}$ https://www.youtube.com/watch?v=wMH0e8kIZtE
} 
Los programas de edición y composición como AfterEffects, Combustion, Cinema $4 \mathrm{D}$ y sus numerosas posibilidades permiten visualizar efectos melódicos o rítmicos de la música de base electrónica y de sus técnicas de masterización digital, como casi toda la que se produce en el panorama musical contemporáneo. Plugins como el Audio Analisys, del software VDMX, vinculan el material visual a parámetros sonoros de manera automática, a través del análisis algorítmico de sus características sonoras: este efecto permite asociar un parámetro visual (color, corte de plano, modo de capas...) para que sea reactivo al beat o a cualquier incidencia rítmica de un instrumento componente (teclado, batería, guitarra, etc.) de la línea musical. Este recurso, que podría describirse como una especie de scratching visual, se encuentra presente en numerosos ejemplos de vídeos en los últimos años como recurso de texturización visual.

En el caso de Telephone ${ }^{2}$, la analogía música/efecto visual parte de los melismas vocales. En la banda musical, la voz es tratada como objeto sonoro, con un efecto de síntesis granular que permite unir fragmentos de sonido muy cortos, cuya longitud oscila entre 5 y 100 milisegundos. La complejidad del sonido granular depende de la cantidad de datos de control que se le dan al generador de granos. En este caso se repite 12 veces por segundo. Su analogía visual parte de repeticiones de acciones cortas en forma de brevísimos flashbacks, que provocan una repetición de acciones corporales como movimientos de extremidades o de cabeza. Este recurso resulta una analogía del sampling, al que se añade un bucle similar al del tratamiento vocal. El efecto de edición descrito trata de imitarlo.

Con un efecto similar puede caracterizarse la realización del vídeo $7-11^{3}$, de Beyoncé, tal como se aprecia en su primer minuto de duración: la edición de microplanos sobre el mismo encuadre permite un efecto minimalista en seguimiento de los riffs vocales.

Otras formas de combinar los tempos cambiantes de música y edición resuenan en Dirty Picture 4 , de Taio Cruz: los planos adelante y atrás con ciertos efectos de cámara rápida intentan visualizar los accelerando y diminuendo musicales, que imitan los scratching del trabajo musical de los discjockeys.

En Wasted time $e^{5}$ de I/O se utilizan dos procesos vocales: un eco y una síntesis granular que complica el melisma. Visualmente se incluye en este

\footnotetext{
${ }^{2}$ https://www.youtube.com/watch?v=EVBsypHzF3U

${ }^{3}$ https://www.youtube.com/watch?v=k4YRWT_Aldo

${ }^{4} \mathrm{https}$ ://www.youtube.com/watch? $\mathrm{v}=\mathrm{RgnXl7fz0Bc}$

${ }^{5}$ https://www.youtube.com/watch? $\mathrm{v}=\mathrm{gU} 7$ fripmU-s
} 
caso una multitud de planos compuestos de pocos frames, que actúan a modo de flashes visuales. El efecto dificulta la visión del contenido del plano e impone relevancia al fenómeno de la edición.

En All night y otros vídeos (del álbum Lemonade, de Beyonce) ${ }^{6}$ se descubre un complejo diseño sonoro, compuesto por respiraciones, gotas de agua, y un universo de acciones microscópicas que permiten dirigir la atención del espectador hacia diferentes sitios del encuadre, $y$ hacia acciones en diferentes planos en profundidad.

\section{Imitación visual}

De igual manera, puede producirse una modulación audiovisual de parámetros o texturas musicales. Working ${ }^{7}$, para Wainrm Myth, trata de visualizar los toques de un instrumento de percusión de ascendencia asiática a través de un efecto o plugin que imita la técnica origami y que permite una especie de collage visual sobre la composición espacial de la imagen. Flaw ${ }^{8}$, de Noah (Takcom, alias Takafumi Tsuchiya), utiliza VFX, fotogrametría y gráficos CGI para modificar objetos y construir formas que visualizan pasajes musicales o partes de la música, de naturaleza electrónica.

Las nuevas técnicas fotográficas de registro (cámaras ultralentas) permiten transportar a visuales muchas de las posibilidades musicales de los instrumentos, la edición y postproducción de sonido digital: técnicas como el slow motion o el timelapse se vienen aplicando a la realización de videoclips. Unconditional rebel ${ }^{9}$, de Siska, visualiza la línea de percusión a través de un plano secuencia en un travelling lateral grabado durante 5 segundos con una cámara ultralenta Phantom 4K, que graba 1000 frames por segundo.

Del mismo modo, en Turn down for what ${ }^{10}$ (Dj Snake y Lil Jon, 2014) algunos efectos musicales de eco y composiciones melódicas del sintetizador en bucle, son visualizadas mediante el temblor corporal de los personajes, que se contagia a la realización a través del movimiento trémulo de desencuadre rápido de la cámara. Mediante la caída de una planta a otra, se produce correspondencia sintagmática entre las secciones de la canción y la evolución de la mínima acción narrativa.

\footnotetext{
${ }^{6}$ https://www.youtube.com/watch?v=gM89Q5Eng_M\&list=PLxKHVMqMZqUSPF11Ghs0KqDf OGhB9Vw5E\&index $=1$

${ }^{7}$ https://www.youtube.com/watch?v=RoYilekwxIk

${ }^{8}$ https://www.youtube.com/watch?v=1OPPl_liDGU

${ }^{9} \mathrm{https} / /$ www.youtube.com/watch?v=0lkIMZ6v6qU

${ }^{10} \mathrm{https} / / /$ www.youtube.com/watch?v=HMUDVMiITOU
} 

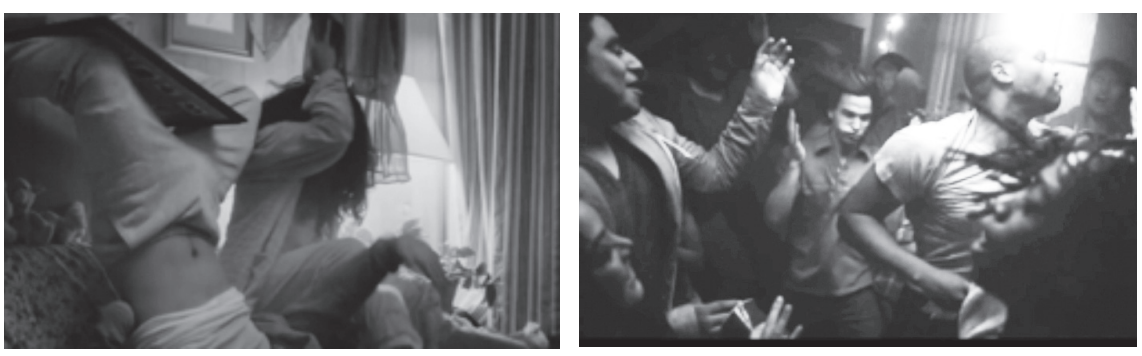

Figuras 2-3. Turn down for what ${ }^{11}$ (Dj Snake, Lil Jon, 2014).

En Straight \& Arrow, de FaltyDL"12 (2012), Daito Manabe crea una "sinfonía de dedos" uniendo un grupo de personas cuyas partes del cuerpo se mueven al ritmo de la música a través de una serie de sensores que, gracias a un software de VJ, disparan una señal sincronizada a determinados elementos de la melodía. Proyectos como este, sin embargo, se encuadran más en obras de raíz electrónica y digital, y, por tanto, están más cercanas a la experimentación desde las artes visuales.

Estas técnicas digitales tienen como consecuencia una revitalización del segmento performance del videoclip contemporáneo. En Oceans $\mathrm{More}^{13}$, de John Lindsay, se descompone visualmente la figura corporal para establecer una analogía con los riffs de guitarra y de igual manera en London Grammar en Wasting my Young Years ${ }^{14}$ ocurre en notas sostenidas de guitarra y de caja de la batería, durante las estrofas. El efecto, similar al bullet time, se crea con 625 cámaras caseras expuestas al mismo tiempo que producen ese temblor visual ${ }^{15}$.

Esta especie de modulación tiene en la danza un recurso de experimentación, aprovechando la función de psicomotricidad corporal de baile que se contempla, mucho más si ello se acompaña del movimiento de cámara y edición básica en el beat (Damasio, 2007; Hagerdoorn, 2010). En el caso de Saint Claude $^{16}$, de Christine and the Machines (2015), el cuerpo se tuerce, se levanta y se estira para modular algunos momentos musicales durante la interpretación de la artista. Este morphing corporal bajo analogía musical

\footnotetext{
${ }^{11}$ https://www.youtube.com/watch?v=HMUDVMiITOU

${ }^{12}$ https://www.youtube.com/watch?v=BREp7HYxJPw

${ }^{13} \mathrm{https} / / /$ vimeo.com/48086981

${ }^{14}$ vimeo.com/155534916

${ }^{15}$ Puede visionarse el fragmento del minuto 1 a 1:30, por ejemplo.

${ }^{16} \mathrm{https}: / /$ www.youtube.com/watch?v=ZzFYmz2lfT4
} 
es coherente con la fluidez de identidades contemporáneas y, según Sobchack (2000), "genera una metafísica del proceso, que consiste menos en ser que en llegar a ser" (p. 8), propio de esta construcción identitaria del artista musical en transformación constante, como define Goodwin con su concepto del texto-estrella.

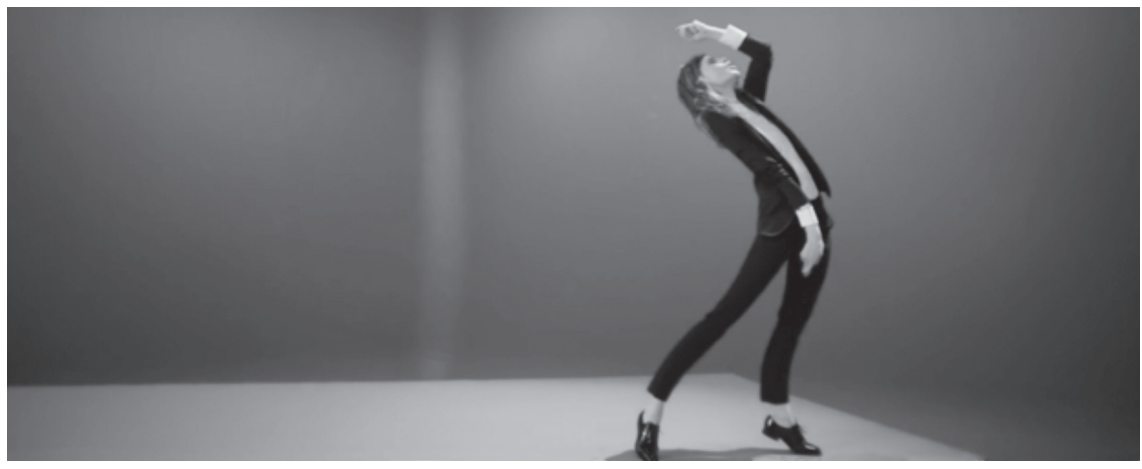

Figura 4. Saint Claude (Christine and the Machines, 2015).

\section{Elementos gráficos textuales}

Otra forma de interconectar lo sonoro y lo visual, mediante decisiones de realización, son las alusiones directas en la banda visual respecto a lo sonoro. La relación de lo visual con las letras de las canciones es controvertida y de compleja categorización (Vernallis, 2002). Su aprovechamiento visual tiene que ver con su naturaleza textual, como ya apuntó Goodwin con la idea de que la disyunción y la amplificación son las fórmulas de relación de letra e imagen: pocos videoclips siguen de manera continua la historia o discurso contado por la letra (Goodwin, 1992, p. 65), es decir, ilustran simplemente la canción. Son los videoclips de una narratividad más clásica los que se apoyan preferentemente en esta conexión musicovisual entre letra/ discurso visual, aunque no suele producirse un seguimiento lineal de su significado, que cerraría demasiado el sentido de la canción.

Por lo tanto, el texto, en cuanto valor gráfico, puede resultar una estrategia útil para crear puntos de sincronía entre banda imagen y banda sonido. Existen algunos ejemplos que permiten seguir afirmando que el videoclip cuida especialmente ciertos fragmentos de las letras como el título o el gancho de la letra (the hook), de los que suele proceder la idea creativa 
que desarrollará la puesta en escena del vídeo. En Bienvenue chez moi ${ }^{17}$, de Stromae, se realiza una presentación visual de su casa mientras se incrustan ciertos textos que coinciden con frases o versos de la letra de la canción.

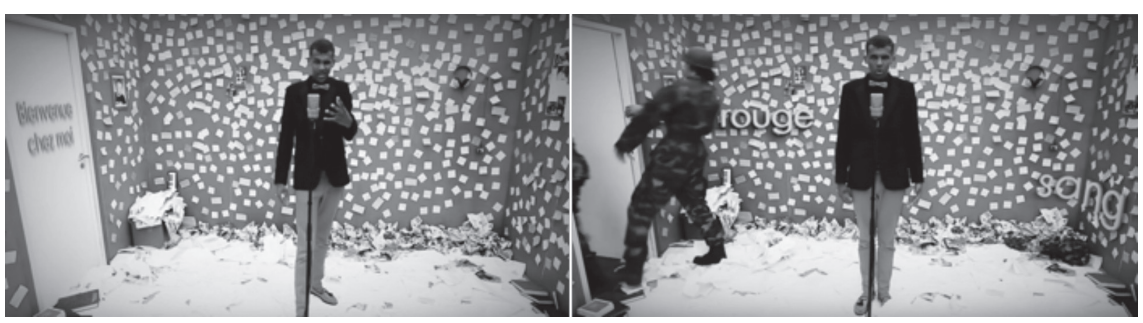

Figura 5. Bienvenue chez moi (Stromae, 2010).

El realizador cuenta con fórmulas estructurales o sintagmáticas para generar síncresis: el modo en que la segmentación de la imagen (normalmente en diversas puestas en escena o acciones) "secunda" la segmentación musical (estrofa/estribillo/estrofa/estribillo/puente/estribillo final, como estructura básica).

En este caso, la transición entre secciones suele hacerse coincidir y se subraya mediante un cambio de plano, preferentemente, u otros tipos de transición, como fundidos a negro y blanco o encadenados. Con frecuencia, los planos de entrada a sección -sobre todo, a las estrofas- contienen un elemento diferenciador: mayor duración, estatismo frente a movimiento, o viceversa.

Esta interesante forma de plantear un videoclip conlleva habitualmente una diversificación de la puesta en escena en más de una localización a través del denominado videoclip mixto, en su forma performance/conceptual o performance/narrativo. Pertenece a un tipo de videoclip mainstream donde se necesita mostrar físicamente al cantante en su interpretación (lo que se hace en la parte performance), que se adereza con el objetivo de ampliar el imaginario de la canción y el cantante (en otro segmento de tipo conceptual).

En Shake it off ${ }^{18}$, de Taylor Swift, aparece claramente esta división, especialmente entre las secciones de estrofa y estribillo, haciendo coincidir esta cesura con cambios en los tipos de puesta en escena alrededor de un tipo

\footnotetext{
${ }^{17}$ https://www.youtube.com/watch?v=QOQISCLKaKQ

${ }^{18} \mathrm{https} / /$ www.youtube.com/watch?v=nfWlot6h_JM
} 
de danza: el paso del baile urbano al clásico, y después al contemporáneo, puntúa las secciones de la estructura musical.

Otra correspondencia sintagmática podría darse a través del movimiento de cámara a modo de loop, haciendo coincidir su fin/principio con las diferenciaciones de secciones de la canción. Esto crea una estructura visual en sí misma en videoclips tan célebres como Come into my world ${ }^{19}$, de Kylie Minogue, dirigido por Michel Gondry. Este plano secuencia, del que Gondry es un maestro, toma la forma de travelling circular, en combinación con una técnica de composición visual por layers, gracias a un avanzado software de postproducción. Comienzo y fin de estos movimientos crean una estructura visual en bucle que va creciendo en complejidad de puesta en escena y se hace paralela a la duración de las secciones de la canción (correspondencia sintagmática). La multiplicación de la fisicidad de Kilye Minogue en la puesta en escena se hace coincidir con las divisiones estrofa/ estribillo/estrofa/estribillo/puente de la canción y refuerza el protagonismo de la estrella, puntuando el título del tema, que parece resultar una apelación a la participación, a la vivencia urbana en comunidad.

\section{Fragmentación y espacialidad}

Algunos de los videoclips de bandas, como Ok Go, describen esta necesidad de entender la visualización a la que debe aspirar el videoclip como una atención de parámetros tanto rítmicos como estructurales. End Love ${ }^{20} \mathrm{de}$ OK Go, trabaja con diferentes formas de visualización para generar sincronía. Por un lado, se encuentran las correspondencias kinéticas, con múltiples modificaciones de tiempo regular a acelerado o lento en determinados momentos texturales musicales y, por otro lado, acciones básicas del cuerpo (levantamiento de pie o mano, brazo, vuelta completa, desplazamiento) coincidentes con notas musicales, del teclado especialmente.

Esto ya ocurría en Ironic ${ }^{21}$, de Alanis Morrissette (1995), donde el espacio era dividido en cuatro partes complementarias: la multiplicación del cuerpo de la cantante y su diferenciación por colores y por la posición en el encuadre, siguiendo las normas de raccord de dirección y respecto de eje de acción, permitía ir reconstruyendo las frases de las estrofas. En cuanto a ello, el vídeo de OK Go parece querer imitar esta idea mediante técnicas

\footnotetext{
${ }^{19} \mathrm{https}: / /$ www.youtube.com/watch?v=63vqob-MljQ

${ }^{20} \mathrm{https}$ ://www.youtube.com/watch?v=V2fpgpanZAw

${ }^{21}$ https://www.youtube.com/watch? $\mathrm{v}=$ Jne9t8sHpUc
} 
actuales de aceleración y timelapse, aunque sobre un mismo plano, no sobre su edición.

En el caso de los videoclips de Stromae, cantante belga-ruandés, se producen confluencias de modos de interrelación: en Papaoutai ${ }^{22}$ (2013) se establecen distintas fórmulas de correspondencia en diversos niveles (rítmico, estructural, etc.), generando un texto audiovisual complejo, que bien podría actuar a expensas de la canción. En primer lugar, la kinética se asegura por la inclusión de la danza, tal como ha sido descrita anteriormente, aunque destaca por su adaptación a ciertos matices de hibridación estilísticas con los modos africanos. En cuanto a la sintagmática, es significativo que el cambio de sección musical se haga coincidir con la contraposición de momentos particulares de padre/hijo protagonistas frente a su comparación con las familias vecinas. Esto ocupa los dos primeros cambios de estrofa/estribillo. El puente de la canción, con un tempo musical más lento, plantea un momento de calma visual. En cuanto al contenido y dentro de una interpretación de la letra bajo las condiciones de amplificación se plantea la relación paterno-filial (el videoclip se llama Papaoutai, transcripción coloquial de Papa, où étais?, en castellano, Papa, ¿dónde estabas?) en términos de contradicción respecto de todas las familias vecinas, que vienen representadas por diferente tipo de movimiento y baile. El chico protagonista contempla admirado los momentos de relación y echa de menos poder vivirlos con su padre (interpretado por el cantante), inmovilizado como figura de cera. Similarmente en Tous les memes ${ }^{23}$ (2013) se denuncia la homogeneidad de comportamientos de género mediante un uso estable y clásico del movimiento de cámara, el color y la transposición de situaciones. En las imágenes pueden verse momentos de comparación entre actitudes y posiciones corporales definidas por una denuncia en la similitud de comportamientos de hombres y mujeres. Cierto mensaje de transgresión sexual y de género, que contiene la canción, viene reforzado mediante elementos kinéticos: la cámara en movimiento circular que une las posiciones femenina y masculina (minutos 2.56 a 3.13) que sigue melódicamente la canción, los momentos coreográficos donde se contraponen movimientos del personaje masculino y femenino.

\footnotetext{
${ }^{22}$ https://www.youtube.com/watch? $\mathrm{v}=$ oiKj0Z_Xnjc

${ }^{23} \mathrm{https} / / / \mathrm{www}$. youtube.com/watch?v=CAMWdvo71ls
} 

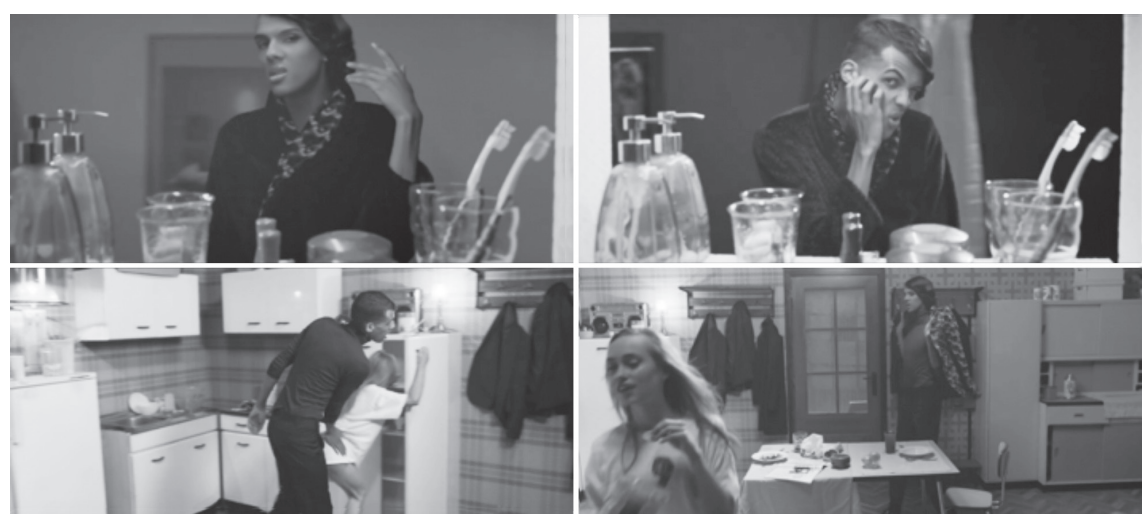

Figura 6. Tous les memes (Stromae, 2013).

Esta dualidad vital y sexual (que también es política) se experimenta visualmente por el movimiento de cámara en steadycam, que permite seguir al personaje (cantante) en sus transformaciones; también por la coreografía, en la que se integran alternancias de perfil, cambios en iluminación y modificación de posturas y gestos. Con ellos, se estructuran acciones a través de estribillos, coros y puente de la canción. La continuidad básica de la canción se representa por el caminar del protagonista seguido por el travelling atrás.

\section{CONCLUSIONES}

Se ha enfocado el cuerpo, en un primer aspecto, como material central de atención de la creación contemporánea y de su estudio en las ciencias humanas y sociales, como campo de experimentación creativa pero también de mercantilización. La relación entre el cuerpo y la imagen, y cómo esta lo ofrece a la mirada del espectador para su goce, define algunos paradigmas de utilización de la tecnología y de su propio desarrollo y evolución hasta una alta sofisticación en postproducción audiovisual. Un segundo aspecto de esta cultura contemporánea conduce a insistir en el crecimiento de la importancia del sonido y de formatos de hibridación audiovisual, de representación de unos medios en otros (sonificación de lo visual, visualización del discurso sonoro): en ellos se moviliza, se contiene, se activa la mirada del espectador para construir experiencias interactivas o de inmersión.

El videoclip musical resulta arquetípico en estos fenómenos: sus creadores llegan desde otros campos de la creación visual como el diseño, la 
animación y las artes plásticas, y en su desarrollo se prueban muchas de las técnicas audiovisuales nuevas de postproducción y tratamiento de imagen.

Con ello, en este panorama el videoclip sigue manteniendo su especificidad de formato audiovisual con objetivo promocional, con la aplicación de técnicas como las analizadas: la visualización diferenciada -el posicionamiento- del cuerpo, performance, del artista en fórmulas híbridas, con materiales altamente postproducidos, supone su principal rasgo. Los ejemplos del corpus trabajado delatan que la imagen del cuerpo adopta diversas estrategias para su visualización, basadas en la capacidad de modulación del mismo como señal. La tecnología de tratamiento y postproducción audiovisual se alían en este objetivo de construcción de una narrativa personal mediante su cuerpo, para estos artistas/músicos.

En el análisis realizado de este corpus de videoclips se han discutido operaciones de visualización de los parámetros musicales (ritmo, textura, frecuencia, etc.) y se ha constatado el uso de la parte performance, donde aparece el cuerpo físico del cantante como contenedor, como lugar donde mejor se visualiza la música mediante fórmulas de síncresis variadas: en analogías de audiovisualización de los rasgos musicales y en operaciones que suponen una imitación visual de sus rasgos. Mediante estas, el cuerpo se fragmenta y espacializa a través de recursos de realización relacionados con el tratamiento/composición de imagen (software y técnicas avanzadas de grabación y edición). Con ello, este soporte físico - corporal muta y es revisitado por las técnicas de la imagen y se ofrece así, mediado, remediado, intensificado y ampliado.

\section{REFERENCIAS}

Aguado, J. C. (2004). Cuerpo humano e imagen corporal. Notas para una antropología de la corporeidad. México D.F.: Universidad Nacional Autónoma de México.

Bernard, M. (1976). El cuerpo. Barcelona: Paidós.

Bjornberg, A. (1994). Structural Relationships of Music and images in Music video. Popular music, 13, 51-74. Consultado el 11 de noviembre de 2015 de https://www.researchgate.net/publication/231852277_Structural_relationships_of_music_and_images_in_music_video

Bourriaud, N. (2009). Postproducción. Buenos Aires: Adriana Hidalgo.

Butler, J. (1993). Cuerpos que importan. Sobre los límites materiales y discursivos del cuerpo. Barcelona: Paidós.

Chion, M. (1993). La audiovisión. Barcelona: Paidos.

Damasio, A. (2007). El error de Descartes. Barcelona: Editorial Dakronos. 
Gaudin, A. (2018). Le clip comme forme d'expression musico-visuelle: pour une esthétique de la relation musique-images. Volume! La revue des musiques populaires 14(2), 97-110. https://doi.org/10.4000/volume.5556

Gaytán, P. (2010). La contribución del estudio del cuerpo y las emociones a las teorías sociológicas de la acción (vs. los estudios culturales). Sociológica 25(72). http://www.scielo.org.mx/scielo.php?script=sci_arttext\&pid $=$ S0187-01732010000100006

Gillet, O., Essid, S., \& Richard, G. (2007). On the Correlation of Automatic Audio and Visual Segmentations of Music Videos. Circuits and Systems for video technologiy, IEEE transactions on 17(3).

Goffman, E. (1981). La presentación de la persona en la vida cotidiana. Buenos Aires: Amorrortu.

Goodwin, A. (1992). Dancing in the distraction factory: Music television and popular culture. Minneapolis: University of Minnesota Press.

Gordillo, I. y Guarinos, V. (2010). Todos los cuerpos. El cuerpo en televisión ccomo obsesión postmoderna. Rosario de Santa Fe: Babel.

Guarinos, V. (2012). Estereotipos y nuevos perfiles de mujer en la canción de consumo. Cuestiones de género 7, 297-314.

Hagerdoorn, I. (2010). Dance, language and the brain. International Journal Arts and Technology 3(2-3), 221-234.

Korsgaard, M. (2012). Creation and Erasure: Music Video as a Signaletic Form of Practice. Journal of Aesthetics \& Culture 4. Consultado el 12 de diciembre de 2015 en http://www.aestheticsandculture.net/index.php/jac/article/ view/18151/22823

Lucerga, M.J. (2004). Ciborgs, forenses y la axila de Sanex. El cuerpo en la sociedad mediática. Tonos. Revista Electrónica de estudios filológicos, 7. http:// www.um.es/tonosdigital/znum7/estudios/icuerpodef.htm

Mairs Slee, S. (2016). Moving the music: Dance, Action, and Embodied identity. En G. Arnold, D. Cookney, K. Fairclough, \& M. Goddard (eds.). Music/ Video: Aesthetics, Media (pp. 147-162). Nueva York: Bloomsbury Academics.

Mendiola, I. y Arpal, J. (2007). Estudios sobre cuerpo, tecnología y cultura. Bilbao: Universidad del País Vasco.

Rafols, R. y Colomer, A. (2006). Diseño audiovisual. Barcelona: Gustavo Gili.

Rodríguez, Á. (1998). La dimensión sonora del lenguaje audiovisual. Barcelona: Paidós.

Sedeño-Valdellos, A. (2012). Música y cultura: propuestas para analizar el cuerpo en el videoclip. Revista de Comunicación Vivat Academia 120, 91-101.

Selva, D. (2012). La visualización de la música en el videoclip. Ámbitos: Revista Andaluza de Comunicación 21, 101-115. Consultado el 23 de diciembre de 2015 en http://www.redalyc.org/articulo.oa?id=16823120006

Shilling, C. (2007). Sociology and the Body: Classical Traditions and New Agendas. The Sociological Review 55(1), 1-18.

Simeon, E. (1992). Programmi narrative e stratificazioni del senso nella musica 
per film. Il caso di "Entr'acte. En R. Dalmonte \& M. Baroni (eds.) Atti di Secondo Convegno Europeo di Analisi Musicale (pp. 389-399). Trento: Universita degli Studi di Trento.

Sobchack, V. (2000). Meta-Morphing:Visual Tranformation and the Culture of Quick-Change. Minneapolis: University of Minnesota Press.

Turner, B. S. (1994). Los avances recientes en la teoría del cuerpo. Reis: Revista Española de Investigaciones Sociológicas 68, Monográfico sobre Perspectivas en Sociología del Cuerpo, 11-39.

Vernallis, C. (1998). The aesthetics of music video: an analysis of Madonna's Cherish. Popular Music 17(2), 153-185.

Vernallis, C. (2002). The functions of lyrics in music video. Journal of Popular studies 14, 11-31.

Vernallis, C. (2004). Experiencing music video. Nueva York: Columbia University Press.

Vernallis, C. (2013). YouTube Aesthetics. Unruly Media: YouTube, Music Video, and the New Digital Cinema (pp. 157-154). Oxford: Oxford University Press.

Villota, G. (2004). Sujeto e imagen-cuerpo: entre la imagen del cuerpo y el cuerpo del espectador. Bilbao: Servicio Editorial de la Universidad del País Vasco. 